\title{
A Concise Stereoselective Route to the Indoline Spiroaminal Framework of Neoxaline and Oxaline
}

\author{
Toshiaki Sunazuka, T atsuya Shirahata, Satoshi T suchiya, Tomoyasu Hir ose, Ryuma \\ Mori, Yoshihiro Harigaya, Isao Kuwajima, and Satoshi Omura -
}

\section{Methods and Material}

General. Unless otherwise noted, all other commercially obtained reagents and solvents were used as received. All reactions were monitored by thin-layer chromatography using E. Merck silica gel 60 F254 pre-coated plates $(0.25 \mathrm{~mm})$. Flash chromatography was performed with indicated solvents using E. Merck silica gel 60 (partical size 0.0400.063). ${ }^{1} \mathrm{H}$ NMR spectra were recorded on JEOL JNM-EX270 (270 MHz), VXR-300 $(300 \mathrm{MHz})$, and ${ }^{13} \mathrm{C}$ NMR spectra were recorded on JEOL JNM-EX270 (67.5 MHz). Chemical shifts are reported relative to internal chloroform $\left({ }^{1} \mathrm{H}, \square=7.26,{ }^{13} \mathrm{C}, \square=77.1\right)$, methanol $\left({ }^{1} \mathrm{H}, \square=3.31,{ }^{13} \mathrm{C}, \square=49.2\right)$, pyridine $\left({ }^{1} \mathrm{H}, \square=8.73,7.58,7.21,{ }^{13} \mathrm{C}, \square=149.9\right.$, $135.5,123.5)$ as indicated. Melting points were obtained on a Y ANAGIMOTO MICRO MELTING APPARATUS and are uncorrected. Infrared spectra were recorded on a Horiba FT-210 spectrometer. High resolution mass spectra were acquired on a JEOL JMS-AX505 HA Mass spectrometer. Low resolution mass spectra were acquired on a JEOL JMS-DX300 Mass spectrometer .

\section{Preparative Pr ocedures.}

(2R)-2-Hydroxy-3 -(1H-indol-3-yl)propionic acid methyl ester ( 6)

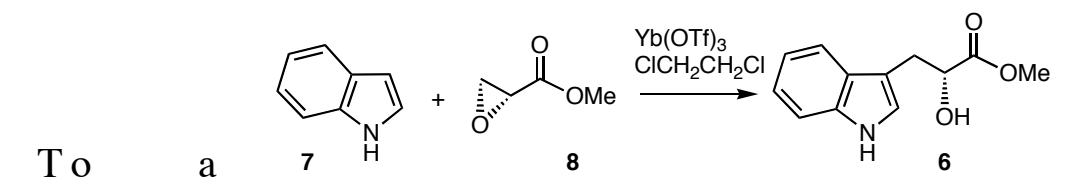

$(1.14 \mathrm{~g}, 9.74 \mathrm{mmol})$ and methyl $(2 R)$ - 2,3-epoxypropanoate 8 (498 $\mathrm{mg}, 4.87 \mathrm{mmol})$ in 1,2-dichloroethane $(9.8 \mathrm{~mL})$ was added $\mathrm{Yb}(\mathrm{OTf})_{3}(303 \mathrm{mg}, 0.487 \mathrm{mmol})$ at room temprature under Ar. The mixture was warmed up to $80^{\circ} \mathrm{C}$ and stirred for $1 \mathrm{hr} 15 \mathrm{~min}$ and then cooled to room temprature. The reaction mixture was quenched with sat. aq. $\mathrm{Na}_{2} \mathrm{CO}_{3}(5 \mathrm{~mL})$, and added $1 \mathrm{~N} \mathrm{HCl}$ aq. $(30 \mathrm{~m} \mathrm{~L}), \mathrm{CHCl}_{3}(30 \mathrm{~mL})$, and two layers were separated. The aqueous layer was extracted with $\mathrm{CHCl}_{3}(30 \mathrm{~mL}$ x 2). The combined organic extracts were washed with brine $(10 \mathrm{~mL} \times 1)$, dried over $\mathrm{Na}_{2} \mathrm{SO}_{4}$, filtered, and evaporated under reduced pressure. Purification by flash chromatography on silica gel (Hexane/EtOAc $=2: 1)$ provided the title compound $\mathbf{6}(818 \mathrm{mg}, 77 \%)$ as a colorless solid. 
$\mathrm{R} f=0.26$ (Hexane/ EtOAc $=1: 1) ;$ m.p. $=65-67^{\circ} \mathrm{C}$; [ $]_{\mathrm{D}}^{22}=+23.4^{\circ}\left(c=1.22\right.$ in $\left.\mathrm{CHCl}_{3}\right) ; \mathrm{IR}$ $(\mathrm{KBr}) \square \mathrm{cm}^{-1}=3344,3054,2949,1732,1620,1437,1352,1180,1093,1009,974,741$, 652; ${ }^{1} \mathrm{H}-\mathrm{NMR}\left(270 \mathrm{MHz}, \mathrm{CDCl}_{3}\right) \square=8.07$ (br-s, $\left.1 \mathrm{H}\right), 7.61(\mathrm{~d}, J=8.2 \mathrm{~Hz}, 1 \mathrm{H}), 7.35(\mathrm{~d}, J$ $=7.6 \mathrm{~Hz}, 1 \mathrm{H}), 7.32-7.10(\mathrm{~m}, 2 \mathrm{H}), 7.10(\mathrm{~s}, 1 \mathrm{H}), 4.53(\mathrm{dd}, J=5.6 \mathrm{~Hz}, 4.6 \mathrm{~Hz}, 1 \mathrm{H}), 3.72(\mathrm{~s}$, $3 \mathrm{H}), 3.31(\mathrm{dd}, J=14.5,4.6 \mathrm{~Hz}, 1 \mathrm{H}), 3.19(\mathrm{dd}, J=14.5,5.6 \mathrm{~Hz}, 1 \mathrm{H}), 2.76(\mathrm{br}-\mathrm{s}, 1 \mathrm{H}) ;{ }^{13} \mathrm{C}-$ NMR $\left(67.5 \mathrm{MHz}, \mathrm{CDCl}_{3}\right) \square=174.7,136.0,127.4,123.3,121.9,119.3,118.6,111.2$, 109.7, 70.7, 52.3, 30.2; HR-MS (FAB, NBA matrix) $m / z: 219.0900[\mathrm{M}]^{+}$, calc for $\mathrm{C}_{12} \mathrm{H}_{13} \mathrm{NO}_{3}: 219.0895[\mathrm{M}]$

(2R)-2-( tert-Butyldimethylsiloxy)-3-(1 H-indol-3-yl)propionic acid methyl ester (I)

To a solution

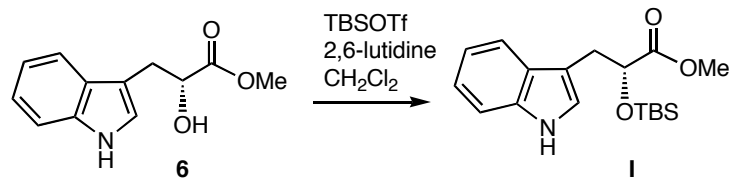

of secondary alcohol $\mathbf{6}$ (2.96 g, $13.5 \mathrm{mmol})$ in $\mathrm{CH}_{2} \mathrm{Cl}_{2}(67 \mathrm{~mL})$ was added 2,6-lutidine (4.73 mL, $40.5 \mathrm{mmol}$ ) and then added TBSOTf $(3.73 \mathrm{~mL}, 16.2 \mathrm{mmol})$ in portions over $10 \mathrm{~min}$ at $0^{\circ} \mathrm{C}$ under $\mathrm{Ar}$. After stirring for $25 \mathrm{~min}$, the solution was quenched by addition of $\mathrm{H}_{2} \mathrm{O}(10 \mathrm{~mL})$. The organic layer was then separated, and the aqueous layer was extracted with $\mathrm{CHCl}_{3}(100$ $\mathrm{mL} \times 3)$. The combined organic extracts were washed with $0.2 \mathrm{~N} \mathrm{HCl}$ aq. $(100 \mathrm{~mL} \times 2)$ and sat. aq. $\mathrm{NaHCO}_{3}\left(100 \mathrm{~mL}\right.$ x 1), dried over $\mathrm{Na}_{2} \mathrm{SO}_{4}$, filtered, and evaporated under reduced pressure. Purification by flash chromatography on silica gel (Hexane/EtOAc $=25: 1)$ provided the title compound I $(4.27 \mathrm{~g}, 94 \%)$ as white solid.

$\mathrm{R} f=0.24$ (Hexane $/$ EtOAc $=5: 1)$; m.p. 90-93 ${ }^{\circ} \mathrm{C} ;[\square]_{\mathrm{D}}{ }^{22}=+6.3^{\circ}\left(c=0.57\right.$ in $\left.\mathrm{CHCl}_{3}\right)$; IR $(\mathrm{KBr}) \square \mathrm{cm}^{-1}=3386,2951,2856,1751,1620,1276,1109,831,787,737 ; \quad{ }^{1} \mathrm{H}-\mathrm{NMR}(270$ $\left.\mathrm{MHz}_{\mathrm{CDCl}}\right) \square=8.02(\mathrm{br}-\mathrm{s}, 1 \mathrm{H}), 7.62(\mathrm{~d}, J=8.3 \mathrm{~Hz}, 1 \mathrm{H}), 7.35(\mathrm{~d}, J=6.3 \mathrm{~Hz}, 1 \mathrm{H})$, 7.33-7.08 (m, 2H) $7.08(\mathrm{~s}, 1 \mathrm{H}), 4.48(\mathrm{dd}, \quad J=7.9,4.6 \mathrm{~Hz}, 1 \mathrm{H}), 3.89(\mathrm{~s}, 3 \mathrm{H}), 3.26(\mathrm{dd}, \quad J=$ 14.7, 4.6 Hz, 1H), 3.10 (dd, $J=14.7,7.9 \mathrm{~Hz}, 1 \mathrm{H}), 0.81(\mathrm{~s}, 9 \mathrm{H}),-0.11(\mathrm{~s}, 3 \mathrm{H}),-0.18$ ( , $3 \mathrm{H}) ;{ }^{13} \mathrm{C}-\mathrm{NMR}\left(67.5 \mathrm{MHz}, \mathrm{CDCl}_{3}\right) \square=174.4,136.5,128.1,123.7,122.3,119.8,119.3$, 111.8, 111.5, 73.3, 52.3, 31.7, 26.1 (3C), 18.7, -5.4, -5.7 ; HR-MS (FAB, NBA, NaI matrix) $m / z: 365.1653[\mathrm{M}+\mathrm{Na}]^{+}$, Calc for $\mathrm{C}_{18} \mathrm{H}_{27} \mathrm{NO}_{3} \mathrm{Na}: 365.1658[\mathrm{M}+\mathrm{Na}]$

(2R)-3-(2-Hydroxy-2- methoxycarbonylethyl)indole-1-carboxylic acid tert-butyl ester (9)

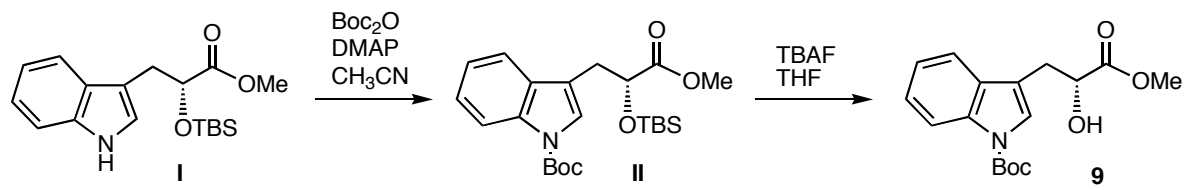

To a

solution of $O$-silylated compound $\mathbf{I}(4.41 \mathrm{~g}, 13.2 \mathrm{mmol})$ in $\mathrm{CH}_{3} \mathrm{CN}(132 \mathrm{~mL})$ was added Boc $_{2} \mathrm{O}(3.6 \mathrm{~mL}, 15.9 \mathrm{mmol})$, DMAP $(161 \mathrm{mg}, 1.32 \mathrm{mmol})$ at room temperature under Ar. After stirring for $1 \mathrm{hr}$, the solution was quenched with $\mathrm{H}_{2} \mathrm{O}(10 \mathrm{~mL})$. Then the organic layer was separated, and the aqueous layer was extracted with $\mathrm{CHCl}_{3}(100 \mathrm{~mL} \times 3)$. The combined organic extracts were washed with sat. aq. $\mathrm{NaCl}(50 \mathrm{~mL}$ x 1), dried over 
$\mathrm{Na}_{2} \mathrm{SO}_{4}$, filtered, and evaporated under reduced pressure. The crude product was used to next reaction without further purification.

Data for the intermediate II: ${ }^{1} \mathrm{H}-\mathrm{NMR}\left(270 \mathrm{MHz}^{\mathrm{CDCl}}{ }_{3}\right) \square=8.15(\mathrm{~d}, J=7.9 \mathrm{~Hz}, 1 \mathrm{H})$, $7.54(\mathrm{~d}, J=8.3 \mathrm{~Hz}, 1 \mathrm{H}), 7.47(\mathrm{~s}, 1 \mathrm{H}), 7.34-7.27(\mathrm{~m}, 2 \mathrm{H}), 4.45(\mathrm{dd}, \quad J=7.9,4.3 \mathrm{~Hz}, 1 \mathrm{H})$, $3.71(\mathrm{~s}, 3 \mathrm{H}), 3.17(\mathrm{dd}, J=14.2,4.3 \mathrm{~Hz}, 1 \mathrm{H}), 3.04(\mathrm{dd}, J=14.2,7.9 \mathrm{~Hz}, 1 \mathrm{H}), 1.66(\mathrm{~s}$, $9 \mathrm{H}), 0.82(\mathrm{~s}, 9 \mathrm{H}),-0.08(\mathrm{~s}, 3 \mathrm{H}),-0.18(\mathrm{~s}, 3 \mathrm{H})$

The crude intermediate II was dissolved in THF $(66.0 \mathrm{~mL})$, and then added TBAF (1.0M sol in THF, $26.4 \mathrm{~mL}, 26.4 \mathrm{mmol}$ ) at room temperature under Ar. After stirring for $10 \mathrm{~min}$, the solution was diluted with $\mathrm{CHCl}_{3}(60 \mathrm{~mL})$, and $\mathrm{H}_{2} \mathrm{O}(10 \mathrm{~mL})$. Then the organic layer was separated, and the aqueous layer was extracted with $\mathrm{CHCl}_{3}(60 \mathrm{~mL} \times 2)$. The combined organic extracts were washed with sat. aq. $\mathrm{NaCl}(10 \mathrm{~mL}$ x 1), dried over $\mathrm{Na}_{2} \mathrm{SO}_{4}$, filtered, and evaporated under pressure. Purification by flash chromatography on silica gel (Hexane/EtOAc $=4: 1)$ provided the title compound $9(3.96 \mathrm{~g}, 94 \%$ 2steps from I) as white solid. $\mathrm{R} f=0.24$ (Hexane/Et OAc $=2: 1)$; m.p. $77-78^{\circ} \mathrm{C}\left(\mathrm{CHCl}_{3}\right) ;[\square]_{\mathrm{D}}{ }^{22}=$ $-3.3^{\circ}\left(c=0.58\right.$ in $\left.\mathrm{CHCl}_{3}\right)$; IR (KBr) $\square \mathrm{cm}^{-1}=3461,3433,2993,1730,1367,1452,1251$, 1155, 1016, 860, 740, 658, $592 ;{ }^{1} \mathrm{H}-\mathrm{NMR}\left(270 \mathrm{MHz}, \mathrm{CDCl}_{3}\right) \square=8.12(\mathrm{~d}, J=7.9 \mathrm{~Hz}$, $1 \mathrm{H}), 7.54(\mathrm{~d}, J=8.3 \mathrm{~Hz}, 1 \mathrm{H}), 7.50(\mathrm{~s}, 1 \mathrm{H}), 7.34-7.28(\mathrm{~m}, 2 \mathrm{H}), 4.53(\mathrm{dd}, \quad J=6.3,4.3 \mathrm{~Hz}$, $1 \mathrm{H}), 3.74(\mathrm{~s}, 3 \mathrm{H}), 3.15(\mathrm{dd}, J=14.5,4.3 \mathrm{~Hz}, 1 \mathrm{H}), 3.10(\mathrm{dd}, J=14.5,6.3 \mathrm{~Hz}, 1 \mathrm{H}), 2.89$ $(\mathrm{d}, J=6.3 \mathrm{~Hz}, 1 \mathrm{H}), 1.66(\mathrm{~s}, 9 \mathrm{H}) ;{ }^{13} \mathrm{C}-\mathrm{NMR}\left(67.5 \mathrm{MHz}, \mathrm{CDCl}_{3}\right) \square=174.5,149.6,135.3$, $130.5,124.3$ (2C), 122.4, 118.9, 115.2, 115.0, 83.5, 70.2, 52.5, 29.9, 28.1 (3C); HR-MS (FAB, NBA matrix) $m / z: 319.1416[\mathrm{M}]^{+}$, Calc for $\mathrm{C}_{17} \mathrm{H}_{21} \mathrm{NO}_{5}: 319.1420[\mathrm{M}]$

(2R,3a $R, 8 \mathrm{a} S)$-3a-Phenylselenyl-2,3,3a,8a-tetrahydrofuro[2,3- $b$ ]indole-2,8dicarboxylic acid 8- tert-butyl ester-2-methyl ester (10) $(2 R, 3 a S, 8 a R)-3 a-P h e n y l s e l e n y l-2,3,3 a, 8 a-t e t r a h y d r o f u r o[2,3-b]$ indole-2,8dicarboxylic acid 8- tert-butyl ester-2-methyl ester ( 11)

To a
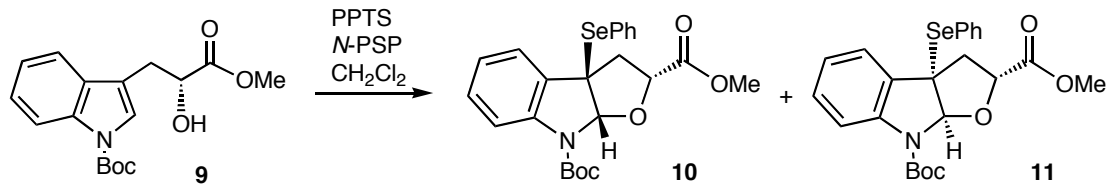

solution of secondary

alcohol 9 (3.59 g, $11.2 \mathrm{mmol})$ in $\mathrm{CH}_{2} \mathrm{Cl}_{2}(112 \mathrm{~mL})$, was added $N$-PSP (5.10 g, 16.8 mmol), PPTS (2.83 g, $11.2 \mathrm{mmol})$ at room temperature under Ar. After stirring for $45 \mathrm{hr}$, the solution was quenched with $\mathrm{H}_{2} \mathrm{O}(20 \mathrm{~mL})$, then the organic layer was separated, and the aqueous layer was extracted with $\mathrm{CHCl}_{3}(150 \mathrm{~mL} \times 2)$. The combined organic extracts were washed with sat. aq. $\mathrm{NaCl}\left(15 \mathrm{~mL}\right.$ x1), dried over $\mathrm{Na}_{2} \mathrm{SO}_{4}$, filtered, and evaporated under reduced pressure. Purification by flash chromatography on silica gel (Hexane/EtOAc $=15: 1)$ provided the title compound $\mathbf{1 0}(2.55 \mathrm{~g}, 49 \%)$ as a yellow oil and the diastereomer $\mathbf{1 1}(2.65 \mathrm{~g}, 50 \%)$ as a yellow oil. The desired compound 10; $\mathrm{R} f=0.29$ (Hexane/EtOAc=3:1); [ $]_{\mathrm{D}}^{22}=-10.8^{\circ}\left(c=2.07\right.$ in $\left.\mathrm{CHCl}_{3}\right)$; IR $(\mathrm{KBr}) \square \mathrm{cm}^{-1}=2978$, 1759, 1714, 1602, 1481, 1392, 1163, 1065, 750, 692; ${ }^{1} \mathrm{H}-\mathrm{NMR}\left(270 \mathrm{MHz}, \mathrm{CDCl}_{3}\right) \square=$ 7.33-6.87 (m, 9H), 6.14 (br-s, 1H), 4.51 (br-d, $J=8.3 \mathrm{~Hz}, 1 \mathrm{H}), 3.16$ (s, 3H), 3.00-2.89 $(\mathrm{m}, 1 \mathrm{H}), 2.74(\mathrm{~d}, J=12.9,8.3 \mathrm{~Hz}, 1 \mathrm{H}), 1.55(\mathrm{~s}, 9 \mathrm{H}) ;{ }^{13} \mathrm{C}-\mathrm{NMR}\left(67.5 \mathrm{MHz}, \mathrm{CDCl}_{3}\right) \square=$ 
$170.8,151.5,142.0,137.0,131.7,129.3$ (2C), 128.9 (2C), 126.2, 126.1, 124.4, 123.1, 114.8, 101.0, 81.4, 76.2, 51.9, 53.3, 42.0, 28.3 (3C); HR-MS (FAB, NBA , NaI matrix) $m / z: 498.0790[\mathrm{M}+\mathrm{Na}]^{+}$, Calc for $\mathrm{C}_{23} \mathrm{H}_{25} \mathrm{NO}_{5} \mathrm{NaSe}: 498.0797[\mathrm{M}+\mathrm{Na}]$; The diastereomer 11; $\mathrm{R} f=0.32($ Hexane/EtOAc $=3: 1) ;[\square]_{\mathrm{D}}^{22}=+100.0^{\circ}\left(c=0.66\right.$ in $\left.\mathrm{CHCl}_{3}\right)$; IR $(\mathrm{KBr}) \square \mathrm{cm}^{-}$ ${ }^{1}=3462,2978,1759,1711,1601,1481,1389,1167,1065,743,692,474 ; \quad{ }^{1} \mathrm{H}-\mathrm{NMR}(270$ $\mathrm{MHz}_{\left., \mathrm{CDCl}_{3}\right)} \mathrm{\square}=7.31-6.87(\mathrm{~m}, 9 \mathrm{H}), 6.28(\mathrm{br}-\mathrm{s}, 1 \mathrm{H}), 4.22(\mathrm{dd}, \quad J=10.2,5.3 \mathrm{~Hz}, 1 \mathrm{H})$, $3.71(\mathrm{~s}, 3 \mathrm{H}), 2.81(\mathrm{dd}, J=12.5,5.3 \mathrm{~Hz}, 1 \mathrm{H}), 2.49(\mathrm{dd}, J=12.5,10.2 \mathrm{~Hz}, 1 \mathrm{H}), 1.52(\mathrm{~s}$, $9 \mathrm{H}) ;{ }^{13} \mathrm{C}-\mathrm{NMR}\left(67.5 \mathrm{MHz}, \mathrm{CDCl}_{3}\right) \mathrm{C}=170.8,151.3,141.0,136.0,132.0,129.2(2 \mathrm{C})$, 128.8 (2C), 126.1 (2C), 126.0, 123.3, 115.0, 100.4, 81.8, 76.1, 52.3, 52.0, 42.8, 28.3 (3C); HR-MS (FAB, NBA, NaI matrix) $m / z: 498.0790[\mathrm{M}+\mathrm{Na}]^{+}$, Calc for $\mathrm{C}_{23} \mathrm{H}_{25} \mathrm{NO}_{5} \mathrm{NaSe}: 498.0797[\mathrm{M}+\mathrm{Na}]$;

$(2 R, 3 a R, 8 a S)-3 a-(1,1-D i m e t h y l-2-p r o p e n y l)-2,3,3 a, 8 a-t e t r a h y d r o-f u r o[2,3-\quad b]$ indole2,8-dicarboxylic acid 8- tert-butyl ester -2-methyl ester (12)

To a
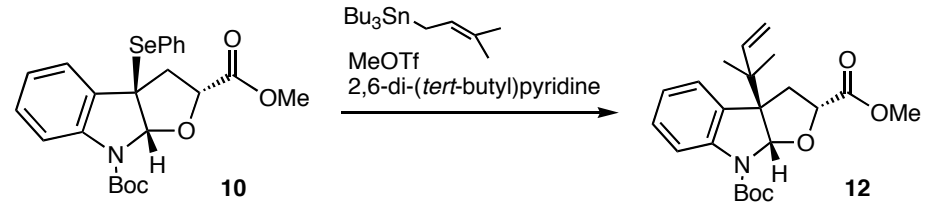

solution of ester $\mathbf{1 0}$

(9.7 g,

20.4 mmol) in

$\mathrm{CH}_{2} \mathrm{Cl}_{2}(204 \mathrm{~mL})$ was added 2,6-di- $t$-butylpyridine $(9.20 \mathrm{~mL}, 40.8 \mathrm{mmol})$, tributyl-(3methyl-2-butenyl)tin $(8.91 \mathrm{~mL}, 26.5 \mathrm{mmol})$ and then added MeOTf $(9.24 \mathrm{~mL}, 81.7$ mmol) under $\mathrm{Ar}$ at $0^{\circ} \mathrm{C}$. After $10 \mathrm{~min}$, the reaction mixture was warmed to room temperature, and stirred for $20 \mathrm{~min}$. The mixture was then warmed to $40{ }^{\circ} \mathrm{C}$, and stirred for $11 \mathrm{hr}$. The solution was quenched with sat. aq. $\mathrm{NaHCO}_{3}(150 \mathrm{~mL})$, the organic layer was separated, and the aqueous layer was extracted with $\mathrm{CHCl}_{3}(300 \mathrm{~mL} \times 2)$. The combined organic extracts were washed with $1.0 \mathrm{~N} \mathrm{NaOH}$ aq. $(100 \mathrm{~mL} \times 1), 1.0 \mathrm{~N} \mathrm{HCl}$ aq. (100 mL x 1), sat. aq. $\mathrm{NaCl}\left(100 \mathrm{~mL}\right.$ x 1), dried over $\mathrm{Na}_{2} \mathrm{SO}_{4}$, filtered, and evaporated under reduced pressure. Purification by flash chromatography on silica gel (Hexane/EtOAc=15:1) provided the title compound $\mathbf{1 2}(4.54 \mathrm{~g}, 57 \%)$ as a yellow oil. $\mathrm{R} f$ $=0.55\left(\right.$ Toluene/EtOAc=5:1); [ []$_{\mathrm{D}}^{22}=+26.5^{\circ}\left(c=1.54\right.$ in $\left.^{2} \mathrm{CHCl}_{3}\right) ; \mathrm{IR}(\mathrm{KBr}) \square \mathrm{cm}^{-1}=$ 3082, 2977, 1760, 1733, 1713, 1601, 1484, 1394, 1165, 1082, 754; ${ }^{1} \mathrm{H}-\mathrm{NMR}(270 \mathrm{MHz}$, $\left.\mathrm{CDCl}_{3}\right) \square=7.80$ (br-m, 1H), 7.22-7.13 (m, 2H), $6.95(\mathrm{t}, J=7.4 \mathrm{~Hz}, 1 \mathrm{H}), 6.07$ (br-s, $\left.1 \mathrm{H}\right)$, $5.91(\mathrm{dd}, J=17.2,10.9 \mathrm{~Hz}, 1 \mathrm{H}), 5.13(\mathrm{~d}, J=10.9 \mathrm{~Hz}, 1 \mathrm{H}), 5.05(\mathrm{~d}, J=17.2 \mathrm{~Hz}, 1 \mathrm{H})$, $4.63(\mathrm{~d}, J=8.6 \mathrm{~Hz}, 1 \mathrm{H}), 3.15(\mathrm{~s}, 3 \mathrm{H}), 2.72(\mathrm{dd}, J=12.5,8.6 \mathrm{~Hz}, 1 \mathrm{H}), 2.56(\mathrm{~d}, J=12.5$ $\mathrm{Hz}, 1 \mathrm{H}), 1.54(\mathrm{~s}, 9 \mathrm{H}), 1.08(\mathrm{~s}, 3 \mathrm{H}), 0.92(\mathrm{~s}, 3 \mathrm{H}) ;{ }^{13} \mathrm{C}-\mathrm{NMR}\left(67.5 \mathrm{MHz}, \mathrm{CDCl}_{3}\right) \square=$ 172.4, 152.9, 143.4 (2C), 131.0, 125.4, 128.8, 122.2, 114.7, 114.3, 96.7, 81.0, 78.2, 60.9, 51.7, 40.3, 37.9, 28.3 (3C), 23.2, 22.4; HR-MS (FAB, NBA matrix) $\quad m / z: 387.2047[\mathrm{M}]^{+}$, Calcd for $\mathrm{C}_{22} \mathrm{H}_{29} \mathrm{NO}_{5}: 387.2046[\mathrm{M}]$

$(2 R, 3 a S, 8 a R)-3 a-(1,1-D i m e t h y l-2-p r o p e n y l)-2,3,3 a, 8 a-t e t r a h y d r o f u r o[2,3-\quad b]$ indole2,8-dicarboxylic acid 8- tert-butyl ester -2-methyl ester (13)
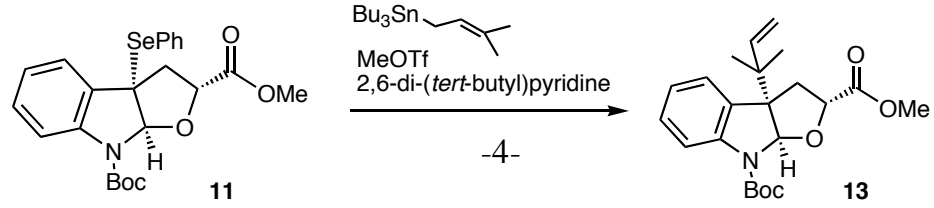
According to the procedure of preparation of compound 12 , the title compound 13 $(958.5 \mathrm{mg}, 56 \%)$ was obtained as a yellow oil from ester $11(2.12 \mathrm{~g}, 4.46 \mathrm{mmol})$. $\mathrm{R} f=$ 0.57 (Toluene/ EtOAc=5:1); [ $\square]_{\mathrm{D}}^{22}=+36.8^{\circ}\left(c=2.36\right.$ in $\left.\mathrm{CHCl}_{3}\right)$; IR $(\mathrm{KBr}) \square \mathrm{cm}^{-1}=3076$, 2976, 1745, 1730, 1716, 1600, 1487, 1394, 1168, 1086, 754; ${ }^{1} \mathrm{H}-\mathrm{NMR}(270 \mathrm{MHz}$, $\left.\mathrm{CDCl}_{3}\right) \square=7.27-7.22(\mathrm{~m}, 3 \mathrm{H}), 7.01(\mathrm{t}, J=7.4 \mathrm{~Hz}, 1 \mathrm{H}), 6.04(\mathrm{br}-\mathrm{s}, 1 \mathrm{H}), 5.84(\mathrm{dd}, \quad J=$ $17.5,10.9 \mathrm{~Hz}, 1 \mathrm{H}), 5.12(\mathrm{~d}, J=10.9 \mathrm{~Hz}, 1 \mathrm{H}), 5.05(\mathrm{~d}, J=17.5 \mathrm{~Hz}, 1 \mathrm{H}), 4.14(\mathrm{dd}, J=$ 10.2, $5.6 \mathrm{~Hz}, 1 \mathrm{H}), 3.72(\mathrm{~s}, 3 \mathrm{H}), 2.44(\mathrm{dd}, J=11.9,10.2 \mathrm{~Hz}, 1 \mathrm{H}), 2.24(\mathrm{dd}, J=11.9,5.6$ $\mathrm{Hz}, 1 \mathrm{H}), 1.58(\mathrm{~s}, 9 \mathrm{H}), 1.07(\mathrm{~s}, 3 \mathrm{H}), 0.99(\mathrm{~s}, 3 \mathrm{H}) ;{ }^{13} \mathrm{C}-\mathrm{NMR}\left(67.5 \mathrm{MHz}, \mathrm{CDCl}_{3}\right) \square=$ $171.4,151.9,143.4,143.0,132.2,129.0,125.0,122.6,115.0,114.3,95.8,81.9,77.2$, 62.1, 52.1, 40.3, 39.0, 28.4 (3C), 23.5, 23.4 ; HR-MS (FAB, NBA matrix) $\mathrm{m} / \mathrm{z}: 387.2043$ $[\mathrm{M}]^{+}$, Calc for $\mathrm{C}_{22} \mathrm{H}_{29} \mathrm{NO}_{5}: 387.2046[\mathrm{M}]$

\section{$(2 R, 3 a R, 8 a S)-3 a-(1,1-D i m e t h y l-2-p r o p e n y l)-3,3 a, 8,8 a-t e t r a h y d r o-2 ~ H-f u r o[2,3-$} $b$ ]indole-2-carboxylic acid methyl ester (5)

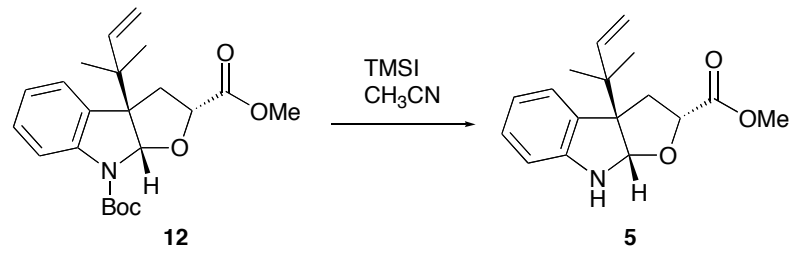

prenylated compound

The

$12(2.00 \mathrm{~g}, 5.18$

$\mathrm{CH}_{3} \mathrm{CN} \quad(51.9$

distilled with $\mathrm{CaH}_{2}$. To a solution of 列 $\mathrm{NaHCO}_{3}(60 \mathrm{~mL})$ at $0^{\circ} \mathrm{C}$, and then stirred for $5 \mathrm{~min}$ at the temperature. The two layers were separated, and the water phase was extracted with $\mathrm{CHCl}_{3}(90 \mathrm{~mL} \times 3)$. The combined organic phase was dried over $\mathrm{Na}_{2} \mathrm{SO}_{4}$, filtered, and evaporated under pressure. Purification by flash chromatography on silica gel (Hexane/EtOAc $=5: 1)$ provided the title compound 5 (1.45 g, 98\%) as a yellow oil. $\mathrm{R} f=0.55($ Hexan e/EtOAc $=1: 1)$; [ $]_{\mathrm{D}}{ }^{22}=$ $+20.9^{\circ}\left(c=1.88\right.$ in $\left.\mathrm{CHCl}_{3}\right)$; IR $(\mathrm{KBr}) \square \mathrm{cm}^{-1}=3458,2963,2930,1759,1713,1601$, 1483, 1464, 1394, 1368, 1162, 1082, 862, 754; ${ }^{1} \mathrm{H}-\mathrm{NMR}\left(270 \mathrm{MHz}, \mathrm{CDCl}_{3}\right) \square=7.10(\mathrm{~d}$, $J=7.6 \mathrm{~Hz}, 1 \mathrm{H}), 7.07(\mathrm{t}, J=7.6 \mathrm{~Hz}, 1 \mathrm{H}), 6.71(\mathrm{t}, J=7.6 \mathrm{~Hz}, 1 \mathrm{H}), 6.60(\mathrm{~d}, J=7.6 \mathrm{~Hz}$, $1 \mathrm{H}), 6.00(\mathrm{dd}, J=17.5,10.9 \mathrm{~Hz}, 1 \mathrm{H}), 5.49(\mathrm{~s}, 1 \mathrm{H}), 5.11(\mathrm{~d}, J=10.9 \mathrm{~Hz}, 1 \mathrm{H}), 5.07(\mathrm{~d}, J=$ $17.5 \mathrm{~Hz}, 1 \mathrm{H}), 4.59(\mathrm{dd}, J=8.6,1.7 \mathrm{~Hz}, 1 \mathrm{H}), 3.23(\mathrm{~s}, 3 \mathrm{H}), 2.73(\mathrm{dd}, \quad J=12.5,8.6 \mathrm{~Hz}$, $1 \mathrm{H}), 2.55(\mathrm{dd}, J=12.5,1.7 \mathrm{~Hz}, 1 \mathrm{H}), 1.12(\mathrm{~s}, 3 \mathrm{H}), 0.99(\mathrm{~s}, 3 \mathrm{H}) ;{ }^{13} \mathrm{C}-\mathrm{NMR}(67.5 \mathrm{MHz}$, $\left.\mathrm{CDCl}_{3}\right) \square=172.5,143.5,143.4,128.9,125.7,122.3,114.8(2 \mathrm{C}), 114.3,96.9,78.3, \quad 60.5$, 51.8, 40.4, 37.9, 23.3, 22.4; HR-MS (FAB, NBA matrix) $\quad m / z: 287.1510[\mathrm{M}]^{+}$, Calc for $\mathrm{C}_{17} \mathrm{H}_{21} \mathrm{NO}_{3}: 287.1521[\mathrm{M}]$
} 
$(2 R, 3 a S, 8 a R)$-3a-(1,1-Dimethyl-2-propenyl)-3,3a,8,8a-tetrahydro-2 $H$-furo[2,3$b$ ]indole-2-carboxylic acid methyl ester (III)

According to preparation of

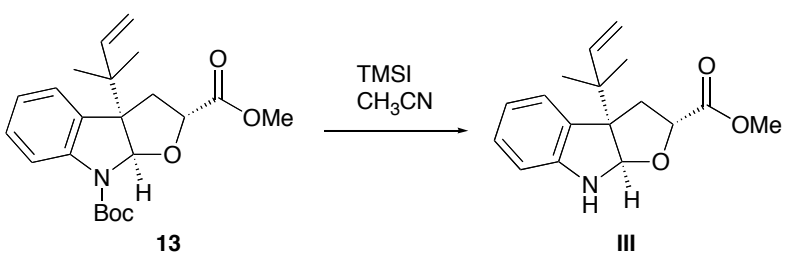

the procedure of 5, III (448 mg, 92\%)

was obtained from $13(659 \mathrm{mg}, 1.70 \mathrm{mmol}) . \mathrm{R} f=0.48($ Hexane/EtOAc $=1: 1) ;[\square]_{\mathrm{D}}{ }^{22}=$ $-64.3^{\circ}\left(c=0.98\right.$ in $\left.\mathrm{CHCl}_{3}\right)$; IR $(\mathrm{KBr}) \square \mathrm{cm}^{-1}=3382,2957,2930,2871,1760,1740$, 1713, 1609, 1485, 1394, 1199, 1094, 918, 744; ${ }^{1} \mathrm{H}-\mathrm{NMR}\left(400 \mathrm{MHz}, \mathrm{CDCl}_{3}\right) \square=7.17$ $(\mathrm{dd}, J=8.0,1.0 \mathrm{~Hz}, 1 \mathrm{H}), 7.09(\mathrm{dt}, J=8.0,1.0 \mathrm{~Hz}, 1 \mathrm{H}), 6.75(\mathrm{dt}, J=8.0,1.0 \mathrm{~Hz}, 1 \mathrm{H})$, $6.58(\mathrm{dd}, J=8.0,1.0 \mathrm{~Hz}, 1 \mathrm{H}), 6.00(\mathrm{dd}, J=17.0,10.4 \mathrm{~Hz}, 1 \mathrm{H}), 5.58(\mathrm{~s}, 1 \mathrm{H}), 5.11(\mathrm{dd}, J$ $=10.4,1.2 \mathrm{~Hz}, 1 \mathrm{H}), 5.05(\mathrm{dd}, J=17.0,1.2 \mathrm{~Hz}, 1 \mathrm{H}), 4.63(\mathrm{br}-\mathrm{s}, 1 \mathrm{H}), 4.22(\mathrm{dd}, \quad J=10.8$, $6.4 \mathrm{~Hz}, 1 \mathrm{H}), 3.73(\mathrm{~s}, 3 \mathrm{H}), 2.42(\mathrm{dd}, J=12.8,10.8 \mathrm{~Hz}, 1 \mathrm{H}), 2.37(\mathrm{dd}, J=12.8,6.4 \mathrm{~Hz}$, $1 \mathrm{H}), 1.11(\mathrm{~s}, 3 \mathrm{H}), 1.01(\mathrm{~s}, 3 \mathrm{H}) ;{ }^{13} \mathrm{C}-\mathrm{NMR}\left(67.5 \mathrm{MHz}, \mathrm{CDCl}_{3}\right) \square=172.0,149.8,143.9$, 129.8, 128.6, 125.2, 118.7, 113.8, 108.5, 96.2, 76.9, 64.5, 52.1, 40.4, 39.6, 23.2, 22.7;

HR-MS (FAB, NBA matrix) m/z: $288.1596[\mathrm{M}+\mathrm{H}]^{+}$, Calcd for $\mathrm{C}_{17} \mathrm{H}_{22} \mathrm{NO}_{3}$ : $288.1600[\mathrm{M}+\mathrm{H}]$

Figure S1. NOE enhancements for prenylated methyl ester III.

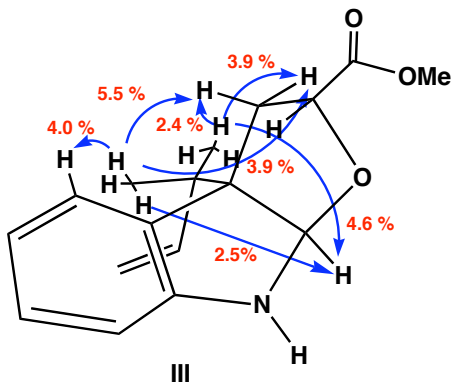

$(2 R, 3 a S, 8 a R)-3 a-(1,1-D i m e t h y l-2-p r o p e n y l)-2,3,3 a, 8 a-t e t r a h y d r o f u r o[2,3-\quad b]$ indole2,8-dicarboxylic acid 8- allyl ester -2-methyl ester (IV)

To the two $(1.45 \mathrm{~g}, \quad 5.05$ and sat. aq.

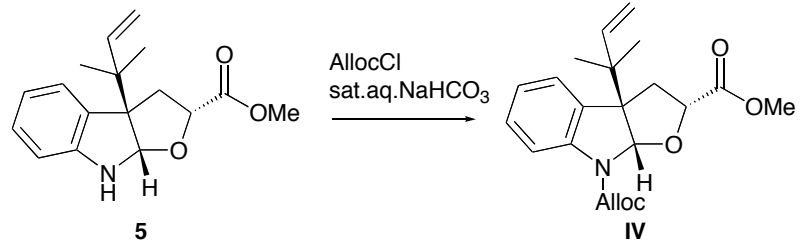

layers solution of $\mathbf{5}$ mmol) in $\mathrm{CH}_{2} \mathrm{Cl}_{2}$ $\mathrm{NaHCO}_{3} \quad(1: 1)$ 
$(252.6 \mathrm{~mL})$ was added AllocCl $(5.34 \mathrm{~mL}, 50.5 \mathrm{mmol})$ at room temprature. After stirring $20 \mathrm{hr}$, the two layers were separated, and the water layer was extracted with $\mathrm{CHCl}{ }_{3}(300$ $\mathrm{mL} \times 2$ ). The combined organic extracts were dried over $\mathrm{Na}_{2} \mathrm{SO}_{4}$, filtered, and evaporated under reduced pressure. Purification by flash chromatography (Hexane/EtOAc=5:1) provided the title compound IV $(1.83 \mathrm{~g}, 98 \%)$. R $f=0.46$ (Hexane/EtOAc=2:1); m.p. $=38-45^{\circ} \mathrm{C}\left(\mathrm{CHCl}_{3}\right)$; [ $]_{\mathrm{D}}{ }^{27}=+34.8^{\circ}\left(c=0.45\right.$ in $\left.\mathrm{CHCl}_{3}\right)$; IR (KBr) $\square \mathrm{cm}^{-1}=2967,1759,1723,1601,1485,1404,1275,1153,1081,933,757,688$; ${ }^{1} \mathrm{H}-\mathrm{NMR}\left(270 \mathrm{MHz}, \mathrm{CDCl}_{3}\right) \square: 7.84(\mathrm{~d}, J=7.3 \mathrm{~Hz}, 1 \mathrm{H}), 7.24(\mathrm{t}, J=6.9 \mathrm{~Hz}, 1 \mathrm{H}), 7.20$ $(\mathrm{d}, J=6.9 \mathrm{~Hz}, 1 \mathrm{H}), 6.98(\mathrm{t}, J=7.3 \mathrm{~Hz}, 1 \mathrm{H}), 6.04(\mathrm{br}-\mathrm{s}, 1 \mathrm{H}), 6.10-5.98,(\mathrm{~m}, 1 \mathrm{H}), 5.92$ (dd, $J=17.2,10.9 \mathrm{~Hz}, 1 \mathrm{H}), 5.42$ (br-d, $J=17.2 \mathrm{~Hz}, 1 \mathrm{H}), 5.27$ (br-d, $J=10.6 \mathrm{~Hz}, 1 \mathrm{H})$, $5.13(\mathrm{dd}, J=10.9,1.0 \mathrm{~Hz}, 1 \mathrm{H}), 5.06(\mathrm{dd}, J=17.2,1.0 \mathrm{~Hz}, 1 \mathrm{H}), 4.89-4.81(\mathrm{~m}, 2 \mathrm{H}), 4.65$ $(\mathrm{dd}, J=8.6,1.0 \mathrm{~Hz}, 1 \mathrm{H}), 3.15(\mathrm{~s}, 3 \mathrm{H}), 2.77(\mathrm{dd}, J=12.9,1.0 \mathrm{~Hz}, 1 \mathrm{H}), 2.57(\mathrm{dd}, J=12.9$, $8.6 \mathrm{~Hz}, 1 \mathrm{H}), 1.08(\mathrm{~s}, 3 \mathrm{H}), 0.96(\mathrm{~s}, 3 \mathrm{H}) ;{ }^{13} \mathrm{C}-\mathrm{NMR}\left(67.5 \mathrm{MHz}, \mathrm{CDCl}_{3}\right) \square=172.1,152.0$, 143.1, 143.1, 132.1, 131.2, 128.9, 125.4, 122.6, 117.4, 114.4, 114.4 , 96.2, 78.3, 65.9, 61.4, 51.7, 40.3, 37.8, 23.1, 22.3; HR-MS (FAB, NBA matrix) $m / z: 371.1731[\mathrm{M}]^{+}$, Calc for $\mathrm{C}_{21} \mathrm{H}_{25} \mathrm{NO}_{5}: 371.1733[\mathrm{M}]$

(2R,3aR,8aS)-2-(Carbamoylmethylcarbamoyl)-3a-(1,1-dimethylpropenyl)-2,3,3a,8atetrahydrofuro[2,3- $b]$ indole-8-carboxylic acid allyl ester (15)

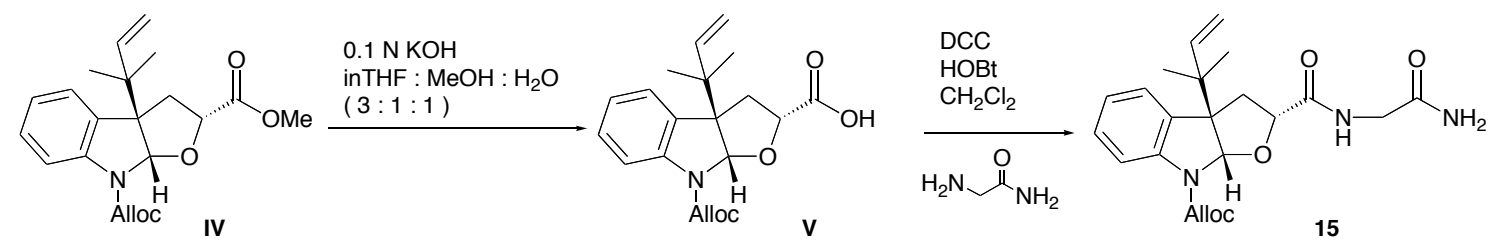

IV $(1.83 \mathrm{~g}, 4.92 \mathrm{mmol})$ was dissolved in $0.1 \mathrm{~N} \mathrm{KOH}$ in $\mathrm{THF}: \mathrm{MeOH}: \mathrm{H}_{2} \mathrm{O}(3: 1: 1)$ solution $(49.2 \mathrm{~mL})$ at room temperature. After stirring $2 \mathrm{hr}$, the mixture was cooled to $0^{\circ} \mathrm{C}$ and added $\mathrm{CHCl}_{3}(20 \mathrm{~mL}), 1.0 \mathrm{~N} \mathrm{HCl}$ aq. $(10 \mathrm{~mL})$, then separated and the water phase was extracted with $\mathrm{CHCl}_{3}(80 \mathrm{~mL} \times 3)$. The combined organic extracts were washed with sat. aq. $\mathrm{NaCl}(60 \mathrm{~mL})$, dried over $\mathrm{Na}_{2} \mathrm{SO}_{4}$, filtered, and evaporated under reduced pressure. The crude product $\mathbf{V}$ was used to next reaction without further purification. The obtained residue was dissolved in $\mathrm{CH}{ }_{2} \mathrm{Cl}_{2}(49.2 \mathrm{~mL})$, and to the solution was added $i$ - $\operatorname{Pr}_{2} \mathrm{NEt}(3.37 \mathrm{~mL}, 34.5 \mathrm{mmol})$, glycineamide $(817 \mathrm{mg}, 7.39 \mathrm{mmol}), \mathrm{HOBt}$ (998 $\mathrm{mg}, 7.39 \mathrm{mmol})$, and DCC $(1.5 \mathrm{~g}, 7.39 \mathrm{mmol})$. After stirring $15.5 \mathrm{hr}$, the solution was evaporated under pressure. To the residue was added $\mathrm{Et}{ }_{2} \mathrm{O}(80 \mathrm{~mL})$, the resultant white precipitate was filtered with Celite, and the solvent was evaporated under reduced pressure. The residue was dissolved in EtOAc $(400 \mathrm{~mL})$, the solution was washed with sat. aq. $\mathrm{NaHCO}_{3}(20 \mathrm{~mL}$ x 3), $1.0 \mathrm{~N} \mathrm{HCl}$ aq. (20mL x 1), sat. aq. $\mathrm{NaCl}(20 \mathrm{~mL}$ x 1), dried over $\mathrm{Na}_{2} \mathrm{SO}_{4}$, filtered, and evaporated under reduced pressure. Purification by flash chromatography $\left(\mathrm{CHCl}_{3} / \mathrm{MeOH}=90: 1\right)$ provided the title compound $\mathbf{1 5}(1.91 \mathrm{~g} 、 94 \%)$ as a yellow powder. $\mathrm{R} f=0.42\left(\mathrm{CHCl}_{3} / \mathrm{MeOH}=15: 1\right)$; m.p. $=58-60^{\circ} \mathrm{C}\left(\mathrm{CHCl}_{3}\right) ;[\square]_{\mathrm{D}}{ }^{27}=$ $+23.2^{\circ}\left(c=1.08\right.$ in $\left.\mathrm{CHCl}_{3}\right)$; IR $(\mathrm{KBr}) \square \mathrm{cm}^{-1}=3385,2969,1711,1680,1524,1484$, 
1404, 1277, 1153, 1075, 919, 757; ${ }^{1} \mathrm{H}-\mathrm{NMR}\left(270 \mathrm{MHz}, \mathrm{CDCl}_{3}\right) \square=7.65$ (br-s, 1H), 7.24$7.19(\mathrm{~m}, 3 \mathrm{H}), 7.01(\mathrm{t}, J=7.6 \mathrm{~Hz}, 1 \mathrm{H}), 6.88(\mathrm{br}-\mathrm{t}, J=5.6 \mathrm{~Hz}, 1 \mathrm{H}), 6.12-5.98(\mathrm{~m}, 1 \mathrm{H})$, $6.08(\mathrm{~s}, 1 \mathrm{H}), 5.88(\mathrm{dd}, J=17.5,10.9 \mathrm{~Hz}, 1 \mathrm{H}), 5.48-5.39(\mathrm{~m}, 1 \mathrm{H}), 5.34-5.29(\mathrm{~m}, 1 \mathrm{H})$, 5.22 (br-s, 1H), 5.13 (dd, $J=10.9,1.0 \mathrm{~Hz}, 1 \mathrm{H}), 5.05$ (dd, $J=17.5,1.0 \mathrm{~Hz}, 1 \mathrm{H}), 4.85$ (d, $J=1.0 \mathrm{~Hz}, 1 \mathrm{H}), 4.83(\mathrm{~d}, J=1.0 \mathrm{~Hz}, 1 \mathrm{H}), 4.58(\mathrm{dd}, J=8.3,3.0 \mathrm{~Hz}, 1 \mathrm{H}), 3.45(\mathrm{dd}, J=$ $16.8,5.6 \mathrm{~Hz}, 1 \mathrm{H}), 3.25(\mathrm{dd}, J=16.8,5.6 \mathrm{~Hz}, 1 \mathrm{H}), 2.78(\mathrm{dd}, J=12.8,8.3 \mathrm{~Hz}, 1 \mathrm{H}), 2.71$ $(\mathrm{dd}, J=12.8,3.0 \mathrm{~Hz}, 1 \mathrm{H}), 1.07(\mathrm{~s}, 3 \mathrm{H}), 0.92(\mathrm{~s}, 3 \mathrm{H}) ;{ }^{13} \mathrm{C}-\mathrm{NMR}\left(67.5 \mathrm{MHz}, \mathrm{CDCl}_{3}\right) \square=$ $172.4,170.6,152.3,143.0,141.7,132.3,132.1,129.0,126.0,123.3,118.3,114.7,114.6$, 96.8, 79.8, 66.6, 61.3, 42.3, 40.4 , 36.3, 23.0, 22.3; HR-MS (FAB, NBA matrix) $\mathrm{m} / \mathrm{z}$ : $414.2033[\mathrm{M}+\mathrm{H}]^{+}$, Calc for $\mathrm{C}_{22} \mathrm{H}_{28} \mathrm{~N}_{3} \mathrm{O}_{5}: 414.2029[\mathrm{M}+\mathrm{H}]$

(2R,3a $R, 8 \mathrm{a} S)-2$-(Carbamoylmethylcarbamoyl)-8-hydro-3a-(1,1-dimethylpropenyl)2,3,3a,8a-tetrahydrofuro[2,3- $b]$-indole (4)

To a
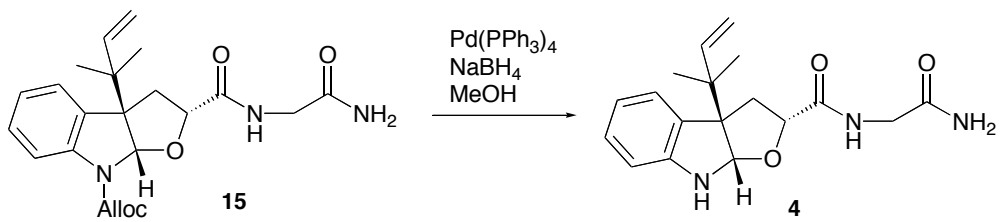

(497.6 mg,

$\mathrm{CH}_{3} \mathrm{OH}(12.0 \mathrm{~mL})$ was added $\mathrm{Pd}\left(\mathrm{PPh}_{3}\right)_{4}$ (13.9 mg, $\left.120 \square \mathrm{mol}\right), \mathrm{NaBH}_{4}(91.1 \mathrm{mg}, 2.41$ $\mathrm{mmol})$ at $0^{\circ} \mathrm{C}$ under Ar. After stirring $15 \mathrm{~min}$, the solution was diluted by $\mathrm{CHCl}{ }_{3}(10$ $\mathrm{mL})$ and then quenched with sat. aq. $\mathrm{NH}_{4} \mathrm{Cl}(10 \mathrm{~mL})$. The mixture was neutralized with sat. aq. $\mathrm{NaHCO}_{3}(10 \mathrm{~mL})$, the organic phase was separated, and the water phase was extracted with $\mathrm{CHCl}_{3} / \mathrm{MeOH}(4: 1)$ solution (30 mL x 12). The combined organic extracts was dried over $\mathrm{Na}_{2} \mathrm{SO}_{4}$, filtered, and evaporated under pressure. Purification by flash chromatography $\left(\mathrm{CHCl}_{3} / \mathrm{MeOH}=80: 1\right)$ provided the title compound $4(367.9 \mathrm{mg}$, 93\%). $\mathrm{R} f=0.26\left(\mathrm{CHCl}_{3} / \mathrm{MeOH}=10: 1\right) ;$ m.p. $=178-181^{\circ} \mathrm{C}\left(\mathrm{CHCl}_{3}\right) ;[\square]_{\mathrm{D}}{ }^{22}=-52.2^{\circ}(c=$ 0.23 in $\left.\mathrm{CHCl}_{3}\right)$; IR (KBr) $\square \mathrm{cm}^{-1}=3371,3199,2985,2962,2362,1697,1637,1533$, 1473, 1411, 1309, 1095, 1020, 997, 885; ${ }^{1} \mathrm{H}-\mathrm{NMR}\left(270 \mathrm{MHz}, \mathrm{CDCl}_{3}\right) \square=7.36$ (br-m, $1 \mathrm{H}), 7.14(\mathrm{~d}, J=7.6 \mathrm{~Hz}, 1 \mathrm{H}), 7.05(\mathrm{dt}, J=7.6,1.0 \mathrm{~Hz}, 1 \mathrm{H}), 6.71(\mathrm{dt}, J=7.5,1.0 \mathrm{~Hz}$, $1 \mathrm{H}), 6.55$ (d, $J=7.6 \mathrm{~Hz}, 1 \mathrm{H}), 5.95$ (dd, $J=17.5,10.9 \mathrm{~Hz}, 1 \mathrm{H}), 5.55$ (br-s, 1H), 5.52 (brs, 1H), 5.15 (br-s, 1H), 5.12 (dd, $J=10.9,1.3 \mathrm{~Hz}, 1 \mathrm{H}), 5.05$ (br-s, 1H), 5.04 (dd, $J=$ $17.5,1.3 \mathrm{~Hz}, 1 \mathrm{H}), 4.54(\mathrm{dd}, J=9.2,1.7 \mathrm{~Hz}, 1 \mathrm{H}), 3.47(\mathrm{dd}, J=16.5,5.6 \mathrm{~Hz}, 1 \mathrm{H}), 3.33$ $(\mathrm{dd}, J=16.5,5.6 \mathrm{~Hz}, 1 \mathrm{H}), 2.76(\mathrm{dd}, J=12.9,9.2 \mathrm{~Hz}, 1 \mathrm{H}), 2.62(\mathrm{dd}, J=12.9,1.7 \mathrm{~Hz}$, 1H), 1.08 (s, 3H), 0.94 (s, 3H); ${ }^{13} \mathrm{C}-\mathrm{NMR}\left(67.5 \mathrm{MHz}, \mathrm{CDCl}_{3}\right) \square=173.8,170.9,148.8$, $143.7,130.3,128.8,125.9,119.2,114.1,109.0,97.7,79.7,63.3,42.7,40.6,37.3,22.8$, 22.5; HR-MS (FAB, NBA matrix) m/z: $329.1719[\mathrm{M}]^{+}$, Calc for $\mathrm{C}_{18} \mathrm{H}_{23} \mathrm{~N}_{3} \mathrm{O}_{3}$ : $329.1739[\mathrm{M}]$ 
(3R,4a $R, 9 \mathrm{a} S)$-4a-(1,1-Dimethyl-2-propenyl)-1-(2-amino-2-oxoethyl)-3-hydroxy-2oxo-2,3,4,4a,9,9a-hexahydropyrido $[2,3-b]$ indole (18)

T o
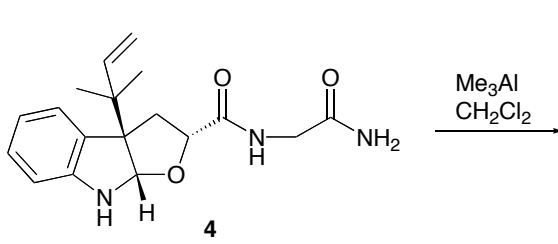

$\mathrm{mg}, 230$ [mol)

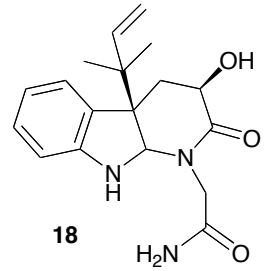

solution of $\mathbf{4}(75.7$

in $\mathrm{CH}_{2} \mathrm{Cl}_{2} \quad(2.30$

$\mathrm{mL})$ was added $\mathrm{Me}_{3} \mathrm{Al}(1.0 \mathrm{M}$ in Hexane $460 \square \mathrm{L}, 460 \square \mathrm{mol})$ at $0^{\circ} \mathrm{C}$ under Ar. After stirring $1 \mathrm{hr}$, the reaction mixture was diluted with $\mathrm{CHCl}_{3}(15 \mathrm{~mL})$, quenched with $30 \%$ Rochelle salt aq. $(10 \mathrm{~mL})$. The two layers were then separated, and the water phase was extracted with $\mathrm{CHCl}_{3}(20 \mathrm{~mL} \times 3)$. The combined organic extracts were washed with sat. aq. $\mathrm{NaCl}(15 \mathrm{~mL} \times 1)$, dried over $\mathrm{Na}_{2} \mathrm{SO}_{4}$, filtered, and evaporated under reduced pressure. Purification by flash chromatography $\left(\mathrm{CHCl}_{3} / \mathrm{MeOH} 100: 1\right)$ provided the title compound $18(57.3 \mathrm{mg}, 76 \%$ ) as a colorless solid. $\mathrm{R} f=0.48$ (developed twice; $\left.\mathrm{CHCl}_{3} / \mathrm{MeOH}=10: 1\right) ;$ m.p. $=81-85^{\circ} \mathrm{C}\left(\mathrm{CHCl}_{3}\right) ;[\square]_{\mathrm{D}}{ }^{24}=-169.0^{\circ}\left(c=0.18\right.$ in $\left.\mathrm{CHCl}_{3}\right) ; \mathrm{IR}$ $(\mathrm{KBr}) \square \mathrm{cm}^{-1}=3401,3348,1670,1649,1606,1487,1471,1315,1259,1155,1132$, 1074, 750; ${ }^{1} \mathrm{H}-\mathrm{NMR}\left(400 \mathrm{MHz}, \mathrm{CDCl}_{3}\right) \square=7.19(\mathrm{~d}, J=8.0 \mathrm{~Hz}, 1 \mathrm{H}), 7.06(\mathrm{dt}, J=8.0$, $1.0 \mathrm{~Hz}, 1 \mathrm{H}), 6.74(\mathrm{dt}, J=8.0,1.0 \mathrm{~Hz}, 1 \mathrm{H}), 6.68(\mathrm{br}-\mathrm{s}, 1 \mathrm{H}), 6.52(\mathrm{~d}, J=8.0 \mathrm{~Hz}, 1 \mathrm{H}), 6.10$ (br-s, 1H), $5.88(\mathrm{dd}, \quad J=17.4,10.8 \mathrm{~Hz}, 1 \mathrm{H}), 5.10(\mathrm{dd}, 10.8,1.2 \mathrm{~Hz}, 1 \mathrm{H}), 5.05(\mathrm{dd}, \quad J=$ $17.4,1.2 \mathrm{~Hz}, 1 \mathrm{H}), 5.02(\mathrm{~d}, 15.6 \mathrm{~Hz}, 1 \mathrm{H}), 4.48(\mathrm{~d}, \quad J=15.6 \mathrm{~Hz}, 1 \mathrm{H}), 3.80(\mathrm{dd}, J=12.6$, $4.4 \mathrm{~Hz}, 1 \mathrm{H}), 3.65(\mathrm{~d}, J=15.6 \mathrm{~Hz}, 1 \mathrm{H}), 2.41(\mathrm{dd}, J=12.6,4.4 \mathrm{~Hz}, 1 \mathrm{H}), 2.07(\mathrm{t}, J=12.6$ $\mathrm{Hz}, 1 \mathrm{H}), 1.11(\mathrm{~s}, 3 \mathrm{H}), 0.95(\mathrm{~s}, 3 \mathrm{H}) ;{ }^{13} \mathrm{C}-\mathrm{NMR}\left(100.5 \mathrm{MHz}, \mathrm{CDCl}_{3}\right) \square=175.9,171.5$, 149.2, 143.5, 129.4, 128.7, 124.9, 118.9, 114.5, 109.0, 79.0, 65.4, 54.8, 50.7, 42.9, 36.6, 22.0, 22.0; HR-MS (FAB, NBA matrix) $m / z: 329.1749[\mathrm{M}]^{+}$, Calc for $\mathrm{C}_{18} \mathrm{H}_{23} \mathrm{~N}_{3} \mathrm{O}_{3}$ : $329.1739[\mathrm{M}]$

(3R,4aR,9aS)-4a-(1,1-Dimethyl-2-propenyl)-1-(2-amino-2-oxoethyl)-3-hydroxy-2oxo-9-oxy-2,3,4,4a,9,9a-hexahydropyrido[2,3- $b$ ]indole (19)

To a solution of
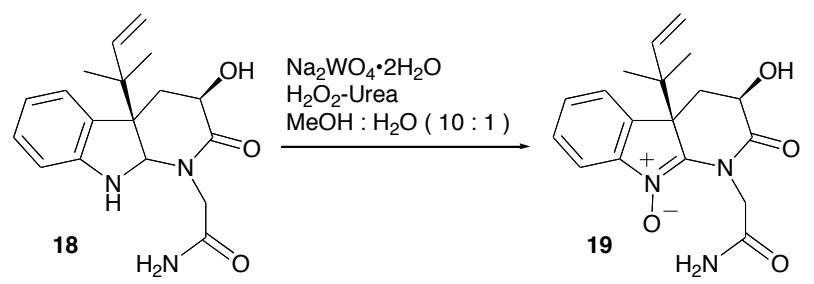

18 (31.8 mg, 96.6 
$\square \mathrm{mol})$ in $\mathrm{MeOH}: \mathrm{H}_{2} \mathrm{O}(10: 1)(967 \square \mathrm{L})$ was added $\mathrm{Na}_{2} \mathrm{WO}_{4} \cdot 2 \mathrm{H}_{2} \mathrm{O}(31.9 \mathrm{mg}, 96.6$ $\square \mathrm{mol}$ ), and $\mathrm{H}_{2} \mathrm{O}_{2} \bullet$ Urea $\left(90.9 \mathrm{mg}, 966 \square \mathrm{mol}\right.$ ) at $0^{\circ} \mathrm{C}$. After stirring $21 \mathrm{hr}$, the mixture was extracted with $\mathrm{CHCl}_{3}(7 \mathrm{~mL} \times 3)$. The combined organic extracts were washed with sat. aq. $\mathrm{NaCl}(10 \mathrm{~mL} \times 1)$, dried over $\mathrm{Na}_{2} \mathrm{SO}_{4}$, filtered, and evaporated under pressure. Purification by TLC (developed twice; $\mathrm{CHCl}_{3} / \mathrm{MeOH}=10: 1$ ) provided the title compound $19(17.0 \mathrm{mg}, 51 \%)$ as a yellow oil. $\mathrm{R} f=0.49\left(\mathrm{CHCl}_{3} / \mathrm{MeOH}=4: 1\right) ;[\square]_{\mathrm{D}}{ }^{26}=+262.4^{\circ}(c=$ 1.50 in $\mathrm{CHCl}_{3}$ ); IR (KBr) $\square \mathrm{cm}^{-1}=3406,2972,1720,1687,1604,1471,1417,1269$, 1140, 1112, 977, 927, 761; ${ }^{1} \mathrm{H}-\mathrm{NMR}\left(270 \mathrm{MHz}^{\mathrm{C}} \mathrm{CDCl}_{3}\right) \square=8.06$ (br-s, 1H), 7.70 (d, J= $7.6 \mathrm{~Hz}), 7.56-7.43(\mathrm{~m}, 3 \mathrm{H}), 5.76(\mathrm{dd}, 17.2,10.9 \mathrm{~Hz}, 1 \mathrm{H}), 5.71(\mathrm{br}-\mathrm{s}, 1 \mathrm{H}), 5.18(\mathrm{~d}, \quad J=$ $10.9 \mathrm{~Hz}, 1 \mathrm{H}), 5.07(\mathrm{~d}, J=17.0 \mathrm{~Hz}, 1 \mathrm{H}), 4.54(\mathrm{~d}, J=14.8 \mathrm{~Hz}, 1 \mathrm{H}), 4.44(\mathrm{~d}, J=14.8 \mathrm{~Hz}$, $1 \mathrm{H}), 4.07(\mathrm{t}, J=9.6 \mathrm{~Hz}, 1 \mathrm{H}), 2.69(\mathrm{dd}, J=13.9,9.6 \mathrm{~Hz}, 1 \mathrm{H}), 2.42(\mathrm{dd}, J=13.9,9.6 \mathrm{~Hz}$, $1 \mathrm{H}), 1.08$ (s, 3H), $0.86(\mathrm{~s}, 3 \mathrm{H}) ;{ }^{13} \mathrm{C}-\mathrm{NMR}\left(67.5 \mathrm{MHz} \mathrm{CDCl}_{3}\right) \square=173.1,169.7,147.6$, 145.6, 141.3, 136.0, 129.1, 128.4, 123.9, 116.4, 114.3, 66.8, 53.9, 47.7, 45.5, 29.4, 22.9, 22.2; HR-MS (FAB, NBA matrix) $m / z: 344.1615[\mathrm{M}+\mathrm{H}]^{+}$, Calc for $\mathrm{C}_{18} \mathrm{H}_{22} \mathrm{~N}_{3} \mathrm{O}_{4}$ : $344.1610[\mathrm{M}+\mathrm{H}]$

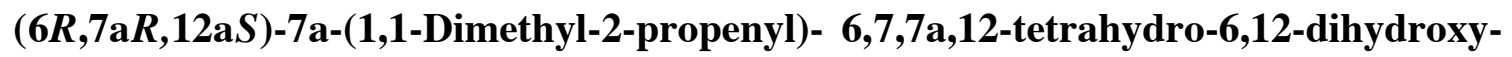
$1 H, 5 H$-imidazo[1',2':1,2]pyrido[2,3-b]indole-2,5(3H)-dione ( 20 )

To a solution

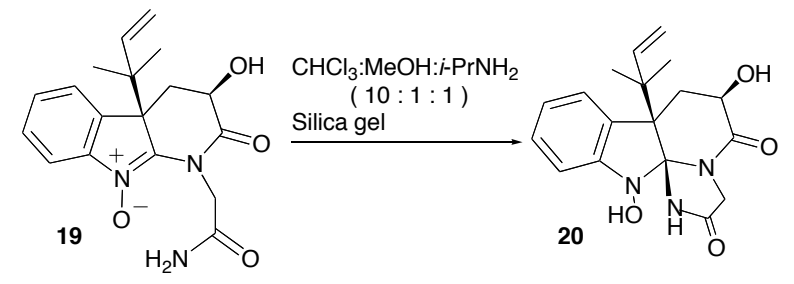

of $19(0.8 \mathrm{mg}, 2.3$ $\mathrm{MeOH}: i-\mathrm{PrNH}_{2}(10$ $\square \mathrm{mol})$ in $\mathrm{CHCl}_{3}$ :

: $1: 1)(800 \square \mathrm{L})$ was added excess silica gel. The solution was stirred for $7.5 \mathrm{hr}$ at $\mathrm{rt}$, and then the solution was filtrated, removed under pressure. Purification by TLC (developed twice; $\left.\mathrm{CHCl}_{3} / \mathrm{MeOH}=7: 1\right)$ provided the title compound $\mathbf{2 0}(0.5 \mathrm{mg}, 63 \%)$ as a yellow oil. $\mathrm{R} f=0.35\left(\mathrm{CHCl}_{3} / \mathrm{MeOH}=10: 1\right) ;$ m.p. $=109-113^{\circ} \mathrm{C}\left(\mathrm{CHCl}_{3}\right) ;[\square]_{\mathrm{D}}{ }^{23}=-59.0^{\circ}(c=0.11 \mathrm{in}$ $\left.\mathrm{CHCl}_{3}\right)$; IR (KBr) cm ${ }^{-1} \square=3473,2972,1724,1668,1591,1475,1369,1292,1216,1124$, 1012, 754; ${ }^{1} \mathrm{H}-\mathrm{NMR}\left(270 \mathrm{MHz}, \mathrm{CD}_{3} \mathrm{OD}\right) \square=7.49$ (d, $\left.J=7.6 \mathrm{~Hz}, 1 \mathrm{H}\right), 7.24(\mathrm{t}, J=7.6$ $\mathrm{Hz}, 1 \mathrm{H}), 6.97(\mathrm{t}, J=7.6 \mathrm{~Hz}, 1 \mathrm{H}), 6.88(\mathrm{~d}, J=7.6 \mathrm{~Hz}, 1 \mathrm{H}), 6.13(\mathrm{br}-\mathrm{m}, 1 \mathrm{H}), 5.17-5.11$ (br-m, 2H), $4.33(\mathrm{dd}, J=12.2,5.3 \mathrm{~Hz}, 1 \mathrm{H}), 4.00(\mathrm{~d}, J=16.5 \mathrm{~Hz}, 1 \mathrm{H}), 3.90(\mathrm{~d}, J=16.5$ $\mathrm{Hz}, 1 \mathrm{H}), 2.30(\mathrm{t}, J=12.2 \mathrm{~Hz}, 1 \mathrm{H}), 2.21(\mathrm{br}-\mathrm{m}, 1 \mathrm{H}), 1.34(\mathrm{~s}, 3 \mathrm{H}), 1.28(\mathrm{~s}, 3 \mathrm{H}) ; \quad{ }^{13} \mathrm{C}-\mathrm{NMR}$ $\left(67.5 \mathrm{MHz} \mathrm{CDCl}_{3}\right) \square=172.6,171.4,146.9,145.2,129.4,126.7,125.2,122.8,113.2$, 111.4, 102.2, 65.6, 53.4, 58.4, 47.0, 36.2, 18.3 (2C); HR-MS (FAB, NBA matrix) $m / z$ : $366.1432[\mathrm{M}+\mathrm{Na}]^{+}$, Calc for $\mathrm{C}_{18} \mathrm{H}_{21} \mathrm{~N}_{3} \mathrm{O}_{4} \mathrm{Na}: 366.1430[\mathrm{M}+\mathrm{Na}]$

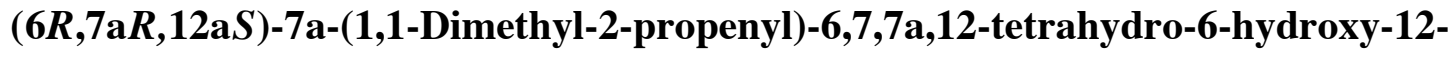
methoxy-1 $H, 5 H$-imidazo[1',2' :1,2]pyrido[2,3-b]indole-2,5(3H)-dione (3)

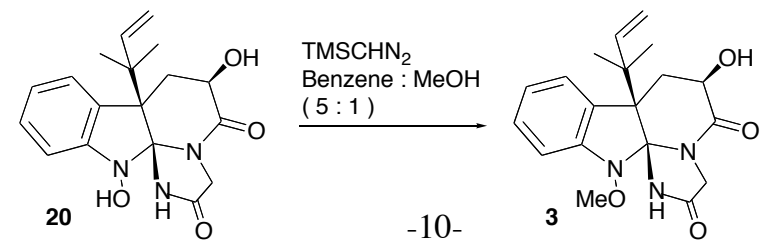


To a solution of 20 (2.3 mg, $6.7 \square \mathrm{mol})$ in Benzene : $\mathrm{MeOH}(5: 1)$ (400 $\square \mathrm{L}$ ) was added $\mathrm{TMSCHN}_{2}(180.0 \square \mathrm{L}, 2.0 \mathrm{M}$ solution in Hexane) at room temperature under Ar. After stirring $29 \mathrm{hr}$, the solution was evaporated under pressure. Purification by Prep. TLC $\left(\mathrm{CHCl}_{3} / \mathrm{MeOH}=10: 1\right)$ provided the title compound $\mathbf{3}(0.7 \mathrm{mg}, 29 \%)$ as yellow oil. $\mathrm{R} f=$ 0.53 (Hexane / EtOAc=1:4); ${ }^{1} \mathrm{H}-\mathrm{NMR}\left(300 \mathrm{MHz}\right.$, pyridine- $\left.{ }_{5}, 80^{\circ} \mathrm{C}\right) \square=7.53(\mathrm{~d}, J=7.8$ $\mathrm{Hz}, 1 \mathrm{H}), 7.24(\mathrm{t}, J=7.8 \mathrm{~Hz}, 1 \mathrm{H}), 6.93(\mathrm{t}, J=7.8 \mathrm{~Hz}, 1 \mathrm{H}), 6.70(\mathrm{dd}, J=7.8,1.5 \mathrm{~Hz}, 1 \mathrm{H})$, 6.42-6.20 (br-m, 1H), 5.15-5.04 (br-m, 2H), 4.68 (d, $J=15.6 \mathrm{~Hz}, 1 \mathrm{H}), 4.60$ (d, $J=15.6$ $\mathrm{Hz}, 1 \mathrm{H}), 4.33$ (br-m, 1H), 3.54 (s, 3H), 2.75 (dd, $J=12.6,4.2 \mathrm{~Hz}, 1 \mathrm{H}), 2.62$ (t, $J=12.6$ $\mathrm{Hz}, 1 \mathrm{H}$ ), 1.39 (br-s, 6H); MS (FAB, NBA matrix) $m / z: 380.1570[\mathrm{M}+\mathrm{Na}]^{+}$, Calc for $\mathrm{C}_{19} \mathrm{H}_{23} \mathrm{~N}_{3} \mathrm{O}_{4} \mathrm{Na}: 380.1586[\mathrm{M}+\mathrm{Na}]$ 